\title{
In memoriam Privatdozent Dr. rer. nat. Wolfgang Marek
}

\author{
In memoriam Privatdozent Dr. rer. nat. Wolfgang Marek
}

Autoren

Institute
K. Rasche ${ }^{1}$, R. Merget $^{2}$

HELIOS Klinikum Wuppertal, Bergisches Lungenzentrum

Institut für Prävention und Arbeitsmedizin der Deutschen Gesetzlichen Unfallversicherung,

Institut der Ruhr-Universität-Bochum (IPA)

\section{Bibliografie}

Dol http://dx.doi.org/ $10.1055 / \mathrm{s}-0030-1256102$ Pneumologie 2011; 65: 123-124 @ Georg Thieme Verlag KG Stuttgart · New York ISSN 0934-8387

\section{Korrespondenzadresse} Prof. Dr. med. K. Rasche HELIOS Klinikum Wuppertal Bergisches Lungenzentrum Direktor der Klinik für Pneumologie, Allergologie, Schlaf- und Beatmungsmedizin Heusnerstr. 40 42283 Wuppertal kurt.rasche@helios-kliniken.de
Wir trauern um unseren langjährigen wissenschaftlichen und klinischen Kollegen, Herrn Privatdozenten Dr. rer. nat. Wolfgang Marek, der am 17. Oktober 2010 im Alter von nur 61 Jahren von uns gegangen ist.

Am 13. März 1949 „tief im Westen“ in Bochum geboren, war Wolfgang Marek ein wirkliches Kind des Ruhrgebiets. Nach seinem Abitur in Bochum im Jahre 1969 nahm er zunächst sein Biologie-Studium an der Westfälischen Wilhelms-Universität in Münster auf, wechselte dann aber zurück in seine Heimat an die noch junge Ruhr-Universität Bochum. Schwerpunkte seines Studiums waren die Fächer Zoologie und Physiologie. Letzteres war dann auch das Grundlagenfach seines späteren wissenschaftlichen Wirkens. Er schloss das Studium im Jahre 1974 erfolgreich als Diplom-Biologe ab. Von 1975 bis 1979 folgte dann seine für ihn als späterer „Angewandter Physiologe“ wahrscheinlich prägendste Zeit als wissenschaftlicher Assistent am Lehrstuhl für Physiologie der Medizinischen Fakultät der Ruhr-Universität Bochum (Direktor: Prof. Dr. Dr. Hans H. Loeschcke). Hier entstand im Jahre 1979 auch seine Dissertationsarbeit mit dem Thema: „Verhalten von Atmung und Kreislauf bei kontinuierlicher und kurzzeitiger elektrischer Reizung von chemosensiblen Afferenzen." Wolfgang Marek blieb bis zum Jahre 1985 wissenschaftlicher Assistent am Lehrstuhl für Physiologie, zunächst noch unter Herrn Professor Loeschcke, dann ab 1982 unter dem neuen Lehrstuhlinhaber, Herrn Professor Dr. Peter Scheid, unter dessen Leitung weitere Arbeiten zur Atmungsregulation von Säugern entstanden.

Mit dem Eintritt in das damalige Silikose-Forschungsinstitut (SFI) der Bergbau-Berufsgenossenschaft Bochum, das spätere Berufsgenossenschaftliche Forschungsinstitut für Arbeitsmedizin (BGFA) und das heutige Institut für Prävention und Arbeitsmedizin der Deutschen Gesetzlichen Unfallversicherung (IPA), vollzog Wolfgang Marek

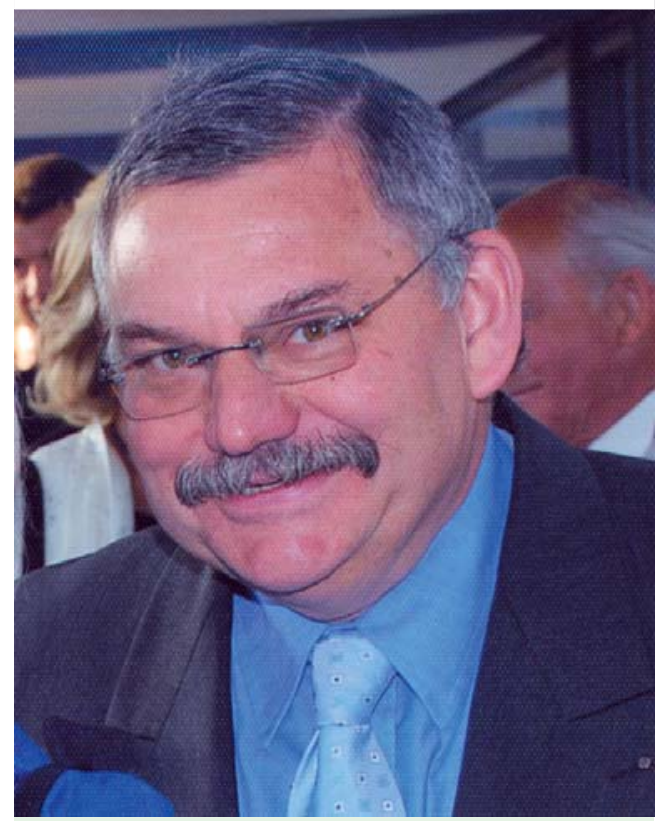

Privatdozent Dr. rer. nat. Wolfgang Marek.

im Jahre 1985 einen für seinen weiteren Werdegang sehr wichtigen Schritt in Richtung Angewandte Physiologie und Klinische Forschung. Unter Leitung von Herrn Professor Dr. Dr. h. c. Wolfgang T. Ulmer war er zunächst als wissenschaftlicher Mitarbeiter, ab 1988 dann als Leiter der Abteilung Pathophysiologie und Toxikologie am BGFA tätig. Sein wissenschaftlicher Schwerpunkt war hier die Grundlagenforschung zur Entstehung berufsbedingter Erkrankungen der Lunge und der Atemwege. Auch trug er zusammen mit Herrn Professor Ulmer wesentlich zur Entwicklung der Lungenfunktionsuntersuchung mittels Bodyplethysmografie bei. Aufgrund des hierbei gewonnenen umfangreichen Erfahrungsschatzes war Wolfgang Marek daher noch im Jahre 2009 Mitverfasser der ersten deutschen Empfehlung zur Bodyplethysmografie und ist auch Mitautor ihrer noch erscheinenden Version in englischer 
Sprache. Es entstanden zahlreiche weitere Publikationen zu Wolfgang Mareks experimentellem und klinischem Forschungsthema, der Pathophysiologie obstruktiver Atemwegserkrankungen.

Im Jahre 1996 wechselte er in die Position des Leiters der Zytologie/Zytometrie im Forschungsinstitut für Frühdiagnose und Therapie des Bronchialkarzinoms an die Klinik für Pneumologie und respiratorische Allergologie der Augusta-Kranken-Anstalt Bochum (Direktor: Prof. Dr. J. A. Nakhosteen) und widmete sich dort thematisch dem Gebiet der Lungenkrebsfrüherkennung durch die automatisierte Sputumzytologie. Hier war er u.a. mit der Durchführung der RIDTELC-Lungen-Studie beschäftigt, die den Stellenwert dieser Methode bei der Krebsfrüherkennung nachweisen konnte. Er beschäftigte sich aber auch weiterhin mit der Methodenentwicklung der Lungenfunktion.

Im Jahre 1998 erfolgte die Habilitation an der Medizinischen Fakultät der Ruhr-Universität Bochum für das Fach Arbeitsphysiologie. 2003 gründete er schließlich das Institut für Arbeitsphysiologie an der Augusta-Kranken-Anstalt, wo er bis zu seinem Tod tätig war. Seine wesentlichen wissenschaftlichen Inhalte waren hier die Lungenfunktionsdiagnostik, insbesondere Sollwerte bei älteren Menschen, Atemgasanalysen und Beurteilungskriterien des 6-Minuten-Gehtests.
Wolfgang Marek war Mitglied in vielen nationalen und internationalen Fachgesellschaften. Ganz besonderen Wert legte er auf die aktive Mitgliedschaft in der Deutschen Gesellschaft für Pneumologie und Beatmungsmedizin sowie der „Bochumer“ Gesellschaft für Lungen- und Atmungsforschung. Er war äußerst aktiv als Organisator der Treffen der Arbeitsgruppe Pathophysiologie der DGP sowie Autor von zahlreichen international hochrangig publizierten Artikeln. Als Hochschullehrer wurde er von den Studenten und seinen von ihm betreuten Doktoranden äußerst geschätzt.

Wolfgang Marek hinterlässt seine ihn stets in seiner Arbeit unterstützende Ehefrau Elisabeth und drei erwachsene Söhne. Uns trifft der Tod von Wolfgang Marek unerwartet. Unser Mitgefühl gilt ganz besonders seiner lieben Familie. Wir alle werden ihn als Kollegen und stets warmherzigen und hilfsbereiten Freund niemals vergessen. Die deutsche Pneumologie verliert mit ihm einen der letzten noch aktiv tätigen Pathophysiologen.
K. Rasche, Wuppertal
R. Merget, Bochum 